\title{
Efficacy of tranexamic acid in reducing blood loss and blood transfusion in idiopathic scoliosis: a systematic review and meta- analysis
}

\author{
Turki Alajmi, Halah Saeed, Khalid Alfaryan, Ahmed Alakeel, Thamer Alfaryan \\ Al-Imam Mohammed Ibn Saud University, College of Medicine, Riyadh, Saudi Arabia \\ Contributions: (I) Conception and design: T Alajmi; (II) Administrative support: None; (III) Provision of study materials or patients: None; (IV) \\ Collection and assembly of data: H Saeed, K Alfaryan; (V) Data analysis and interpretation: A Alakeel; (VI) Manuscript writing: All authors; (VII) \\ Final approval of manuscript: All authors. \\ Correspondence to: Turki Alajmi. Al-Imam Mohammed Ibn Saud Islamic University, Riyadh, Saudi Arabia. Email: turki--aj@hotmail.com.
}

\begin{abstract}
Background: Tranexamic acid is a synthetic lysine-analogue antifibrinolytic that competitively inhibits the activation of plasminogen to plasmin, it is a well-documented blood sparing agent. However, its routine use in idiopathic scoliosis surgery is poorly documented. The objective of this meta-analysis was to determine TXA's efficacy in reducing blood loss and blood transfusion in idiopathic scoliosis surgery.

Methods: Five databases (Medline, PubMed, Web of Science, Embase and The Cochrane Central Register of Controlled Trials) were searched to identify the relevant randomized controlled trials (RCTs), prospective cohort control (PCC), and retrospective controlled trails regarding the TXA efficacy in idiopathic scoliosis surgery. Mean differences (MDs) of blood loss and blood transfusions in TXA-treated group compared to control and/or placebo group were extracted and combined using random-effect meta-analysis model.

Results: A total of seven studies comprising 426 patients were included in the meta-analysis according to the pre-defined selection criteria. TXA-treated group had an overall significantly $(\mathrm{P}<0.005)$ less volume of blood loss [ES $(\mathrm{MD})=727.71 \mathrm{~mL}$; CI, 281.86-1,173.56 mL]. Six studies comprising 346 patients TXAtreated group had an overall significantly $\{\mathrm{P}<0.001$ less transfusion volume $[\mathrm{ES}(\mathrm{MD})=268.30 \mathrm{~mL}$; CI, 105.19-431.44 mL]\}.
\end{abstract}

Conclusions: Patients treated with TXA had a significantly lower blood loss and lower rates of allogeneic blood transfusion than the control group. Further investigation is required regarding the safety of TXA before it can be generalized in the use of idiopathic scoliosis surgery.

Keywords: Bleeding; tranexamic acid; scoliosis; spine; surgery; meta-analysis

Submitted May 05, 2017. Accepted for publication Aug 22, 2017.

doi: 10.21037 /jss.2017.08.17

View this article at: http://dx.doi.org/10.21037/jss.2017.08.17

\section{Introduction}

Idiopathic scoliosis is the commonest type of spinal deformity confronting spinal surgeons (1). Scoliosis represents a disturbance of the well-organized 25 member intercalated series of spinal segments. It is, at times, grossly oversimplified as mere lateral deviation of the spine, when in reality, it is a complex three-dimensional deformity (2). Two-dimensional imaging systems (plain radiographs or $\mathrm{X}$-ray) remain somewhat limiting, and scoliosis is commonly defined as greater than $10^{\circ}$ of lateral deviation of the spine from its central axis (3). Once active intervention is indicated, surgical treatment to achieve solid bony fusion is the treatment of choice (4). It is well known that one of the main concerns regarding patients undergoing major operative procedures is the risk of increased perioperative bleeding (5) and major morbidity and mortality rates are associated with intraoperative blood loss and transfusion, thus the use of antifibrinolytics and other 
measures to control bleeding is crucial. In response to large volumes of blood loss, patients will face an increase in the need of blood transfusion as well as other measures such as intraoperative blood salvage, hemodilution, and controlled hypotension (6). Tranexamic acid is a synthetic lysineanalogue antifibrinolytic that competitively inhibits the activation of plasminogen to plasmin; at high concentrations it non-competitively blocks plasmin, thus, tranexamic acid inhibits the dissolution and degradation of fibrin clots by plasmin (7). It has been implicated in major surgical specialities including cardiac surgery (8), trauma (9) and total knee arthroplasty (10). Over the last decade, the use of pharmacological approach to control blood loss has highly been promising and recommended (11). The use of TXA in spinal surgery has been studied and documented (12), but the literature still lacks strong data studying its effectiveness in major spinal procedures. Scoliosis in particular, adolescent idiopathic scoliosis to be specific has a strong impact on patients undergoing scoliosis correction surgery whether it involves posterior or anterior spinal fusion. The procedure itself is not quite simple, in which the surgeon might have to face many objectives and difficulties whether they are intraoperative or postoperative which might include postoperative infection, spinal cord injury, and blood loss associated with major spinal instrumentation and its corresponding procedures. In a retrospective analysis that involved 340 patients and aimed to predict the operative blood loss during spinal fusion of adolescent idiopathic scoliosis, the estimated blood loss was $907 \pm 775 \mathrm{~mL}$ for posterior spinal fusion, $323 \pm 171 \mathrm{~mL}$ for anterior spinal fusion, and $1,277 \pm 821 \mathrm{~mL}$ for combined procedures (13), which emphasize that the blood loss in spinal fusion to correct adolescent idiopathic scoliosis cannot be neglected nor underestimated. The objective of this meta-analysis is to determine if the antifibrinolytic tranexamic acid is effective in reducing the amount of blood loss and allogenic blood transfusion in patients with idiopathic scoliosis undergoing spinal fusion in order to correct the deformity.

\section{Methods}

\section{Study design}

We conducted a meta-analysis and systematic review according to the predefined guidelines provided by the Cochrane Collaboration. Five databases (PubMed, Medline, Cochrane Central Register of Controlled Trials, Web of Science and Embase) were searched to identify the relevant randomized controlled trials (RCTs), prospective cohort control (PCC), and retrospective control trials regarding the effectiveness of TXA in idiopathic scoliosis surgery.

\section{Search strategy and data source}

Interrelated RCTs, PCC, and retrospective control trials were distinguished in Medline, Embase, PubMed, Web of Science databases and the Cochrane Central Register of Controlled Trials from January 1960 through December 2016. Key words used in the search included "antifibrinolytics", "tranexamic acid”, "cyklokapron", "aprotinin”, "trasylol”, “epsilon aminocaproic acid”, "amicar" and, "spinal curvatures", "kyphosis", "scoliosis", "lordosis", "anterior fusion" "posterior fusion", "randomized controlled trial", "comparative study", "prospective" and "retrospective".

\section{Inclusion and exclusion criteria}

All the studies were included in accordance to all the criteria included mutually based on criteria from (I) to (V) as follows: (I) RCTs, PCC and/or retrospective control; (II) subjects who underwent spinal fusion including cervical, thoracic and lumber scoliosis correction surgery irrespective of anterior or posterior approach; (III) the route of intervention was TXA intravenous [IV] administration in the treatment (experimental) group, accompanied with matched control group; (IV) the primary outcome measures should include one of the following outcomes: volume of total blood loss reported outcomes including intraoperative, postoperative, and total blood loss (primary outcomes); and (V) the outcomes that were described included the number of patients receiving blood transfusion, volumes of transfusion packed cells.

Studies should be excluded if they: (I) had no interventions of which was described above; (II) studies that included patient with a history of coagulation disorder or recent intake of anticoagulation drugs (1 month); (III) patients with severe cardiopulmonary, renal, and hepatic dysfunction; (IV) did not contain any of the outcomes mentioned above; $(\mathrm{V})$ involved procedures in idiopathic scoliosis patient other than primary deformity correction.

\section{Literature search results}

The internet literature search on TXA yielded a total of 98 studies; PubMed ( $\mathrm{n}=25)$; Medline ( $\mathrm{n}=24)$; Cochrane Central Registered Controlled Trials ( $\mathrm{n}=18)$; Embase $(\mathrm{n}=15)$ and Web of Science $(n=16)$. Of these 98 , only 25 articles 


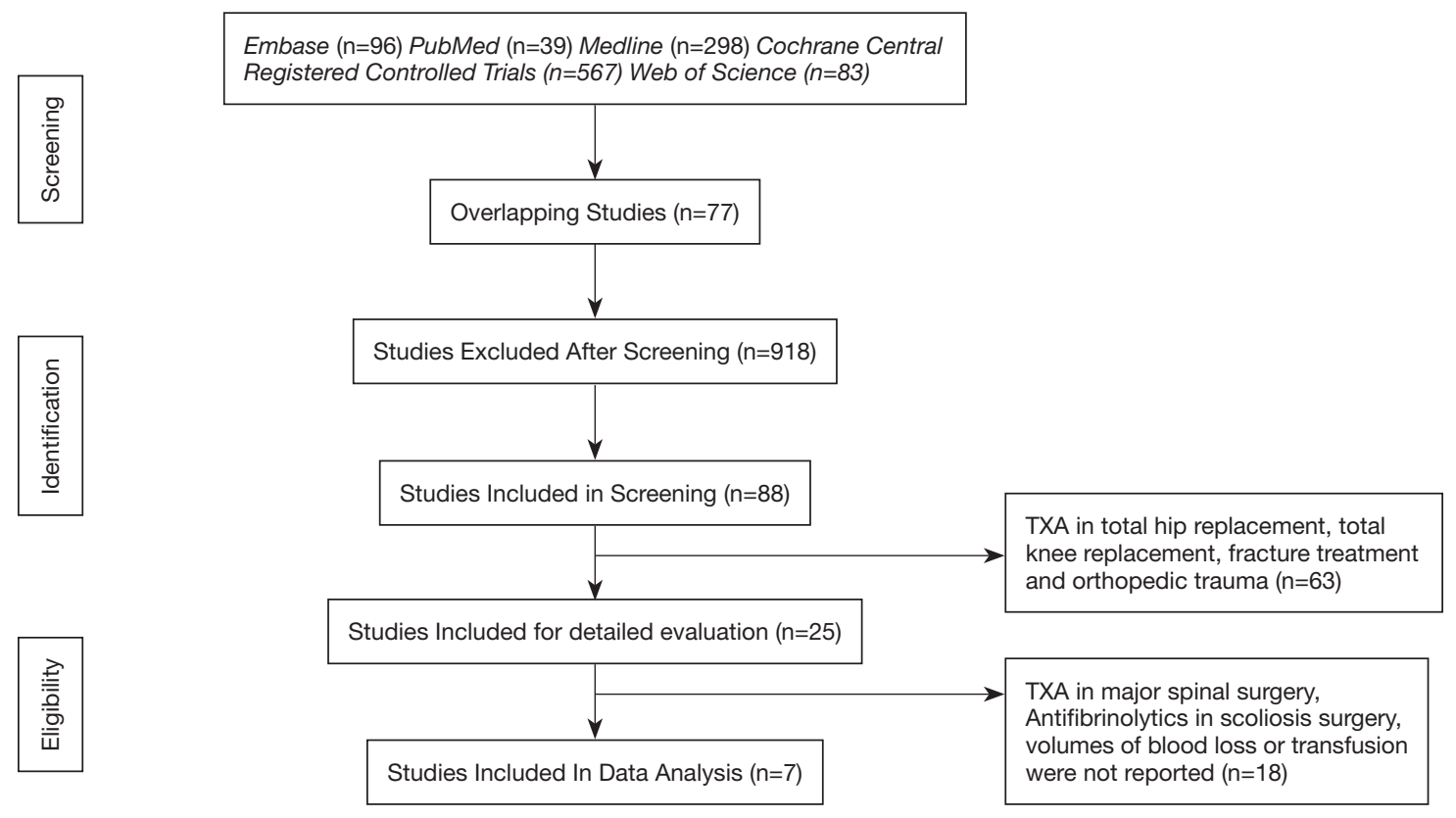

Figure 1 The flow chart showing process of literature review.

were found to be relevant articles on scoliosis corrective surgery regardless of approach. Although all 25 studies were relevant, only 7 were selected for data extractions. The remaining 19 studies did not meet the inclusion criteria and/or did not contain the required information. Figure 1 summarizes the steps of the literature search and review .

\section{Data extraction}

Eligible articles were reviewed independently by two investigators. The titles and abstracts of the references were read. Any disagreement on a controversial study was settled by discussion and consensus with a third author. The collected data included the first authors' name, publication date, region/ethnicity, genotyping method and the total number of cases and controls.

\section{Bias risk assessment}

The included studies were assessed for risk of bias by two independent researchers and a managing reviewer. Methodological domains were graded individually according to (I) study's design; (II) adequacy of the methods; (III) level of accuracy of the measurements of blood volumes; (IV) appropriateness of the statistical tests used for reporting results. The included studies were graded accordingly (I) adequate - methods were reported and appropriate; (II) inadequate-methods were reported but inappropriate; or (III) unclear-methods were not reported. In addition, all efforts were made to ensure the implementations of Cochrane guidelines for literature search.

\section{Statistical analysis}

The effect size [mean differences (MD)] of blood loss and blood transfusions in TXA-treated group compared to control and/or placebo group were extracted and combined using random-effect meta-analysis. Heterogeneity parameters were used to evaluate the variability among studies relative to the overall effects size. Forest-plot was generated to display the range of each study relative to the overall range of the model.

\section{Results}

After full text review of the relevant 25 studies, only 7 qualified studies were used in the meta-analysis for blood loss (14-20).

Additionally, 6 studies (14,15,17-20) were included in the final analysis for blood transfusion. The basic gender and anthropometric characteristics of the studies are involved in this meta-analysis presented in Table 1, Figures 2 and 3. 
Table 1 Basic characteristics of the studies involved in the meta-analysis of the present study

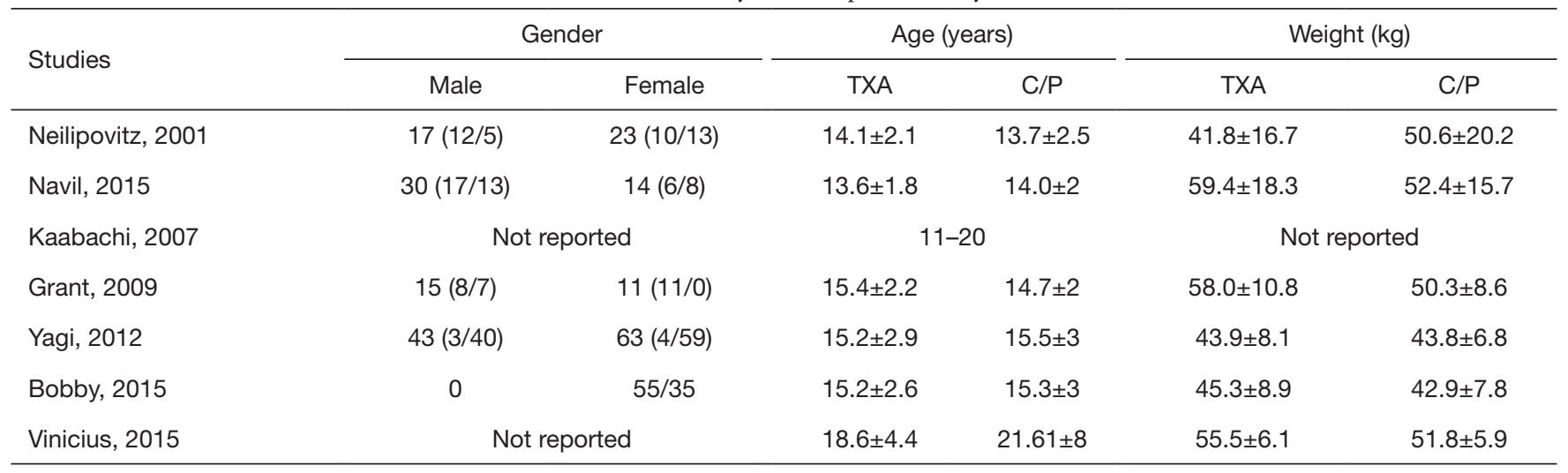

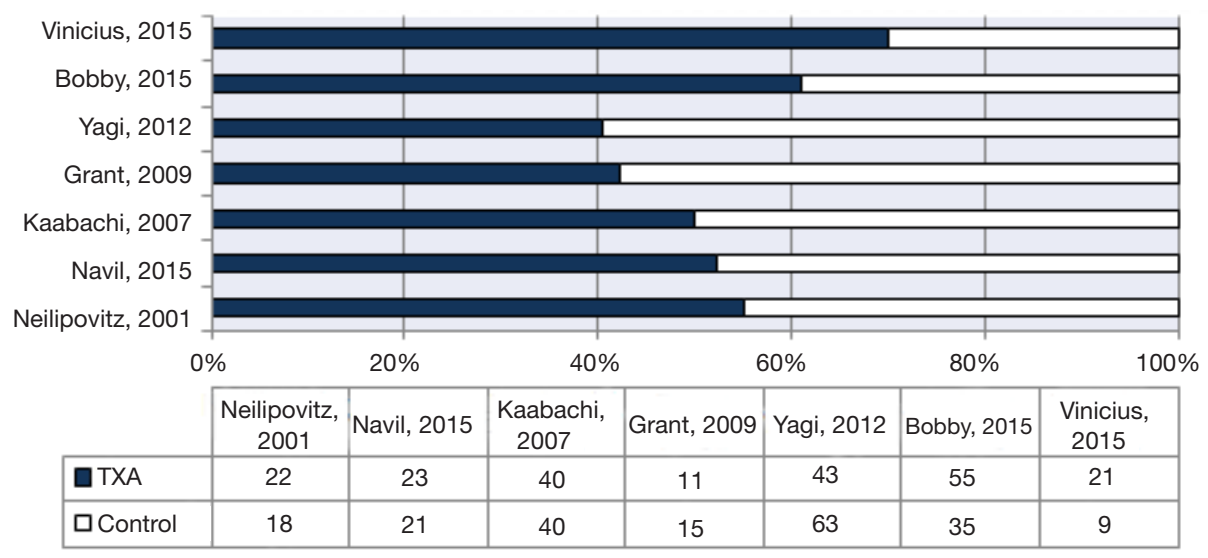

Figure 2 Number of patients in TXA and control groups in each study.

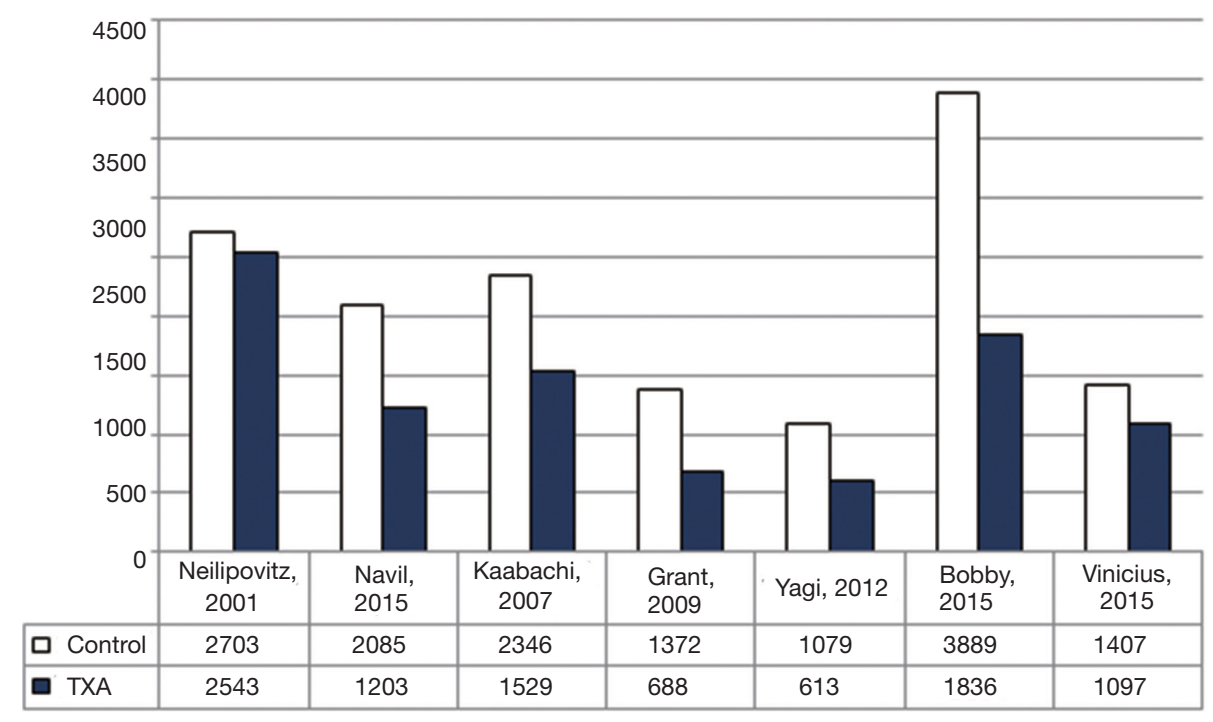

Figure 3 Volume of blood loss in each included study. 
Table 2 Studies profile, effect size, confidence intervals and weight for menstrual blood loss

\begin{tabular}{|c|c|c|c|c|c|c|c|c|}
\hline Studies & \multicolumn{2}{|c|}{ Control } & \multicolumn{2}{|c|}{ TXA } & MD & \multicolumn{2}{|c|}{ Confidence interval } & Weight $(\mathrm{kg})$ \\
\hline Neilipovitz, 2001 & 18 & 2,703 & 22 & 2,453 & 250 & -623.734 & $1,123.734$ & 10.81 \\
\hline Navil, 2005 & 21 & 2,085 & 23 & 1,230 & 855 & 301.848 & $1,408.152$ & 14.40 \\
\hline Kaabachi, 2007 & 40 & 2,346 & 40 & 1,529 & 817 & 374.214 & $1,259.786$ & 15.64 \\
\hline Yagi, 2012 & 63 & 1,079 & 43 & 613 & 466 & 346.818 & 585.182 & 18.26 \\
\hline Bobby, 2015 & 35 & 3,889 & 55 & 1,826 & 2,063 & $1,205.244$ & $2,920.536$ & 10.98 \\
\hline Vinicius, 2015 & 19 & 1,407 & 21 & 1,097 & 310 & 91.979 & 526.421 & 17.72 \\
\hline
\end{tabular}

MBL, menstrual blood loss; MD, mean differences.

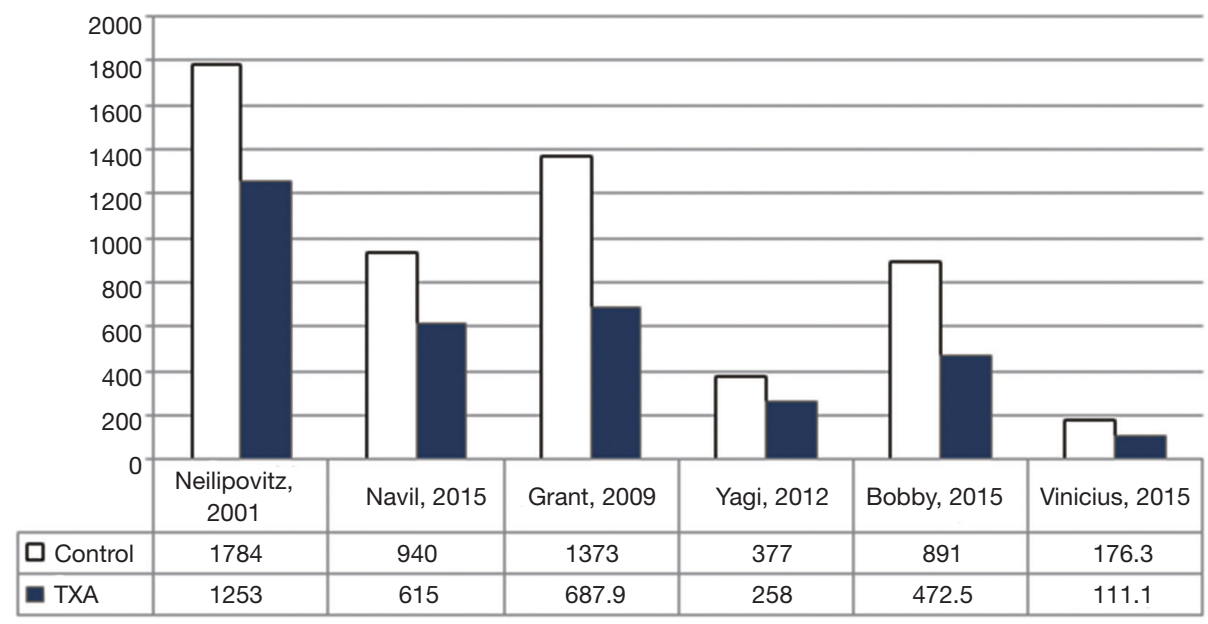

Figure 4 Volume of blood transfusion in each included study.

\section{Meta-analysis for blood loss volume (BLV)}

For intraoperative blood loss, a total of seven studies comprising 426 patients were included in the meta-analysis according to the pre-defined selection criteria, described in the methods section. Table 1 summarized the characteristics and weight effect of the seven studies included for BLV.

As shown in Table 2, all studies' profile for both TXA and control groups included effect size (MD), upper and lower limits of the confidence intervals (CI) and weight. Figure 4 illustrated the mean blood loss in both control and TXA groups. Clearly observed overlap in forest's plot among the CI's for all studies that indicated generalized homogeneity (Figure 5). The overall model results indicated that the effect size of blood volume loss was $727.71 \mathrm{~mL}$ with CI ranged from 281.86 to $1,173.56 \mathrm{~mL}$, which was significant $(\mathrm{P}<0.005)$, implying that the use of TXA caused a substantial reduction in BLV during operation. The model also displays a 95\% CI for the between-studies variance estimate implying perfect overlap, hence, the observed scatter is due to sampling error and the null hypothesis is rejected. It can be concluded that the true effects of TXA in all studies are identical. This was also true as $\mathrm{I}^{2}=90.34 \%$ (Table 3 ) implied more than $90.34 \%$ of the proportion of BLV reduction inducted by TXA treatment effect (Table 3).

\section{Meta-analysis for blood volume transfusion (BVT)}

For post-surgical blood transfusions, a total of six studies comprising 346 patients were included in the meta-analysis according to the pre-defined selection criteria, mentioned in the methods section. Table 4 summarized the characteristics 


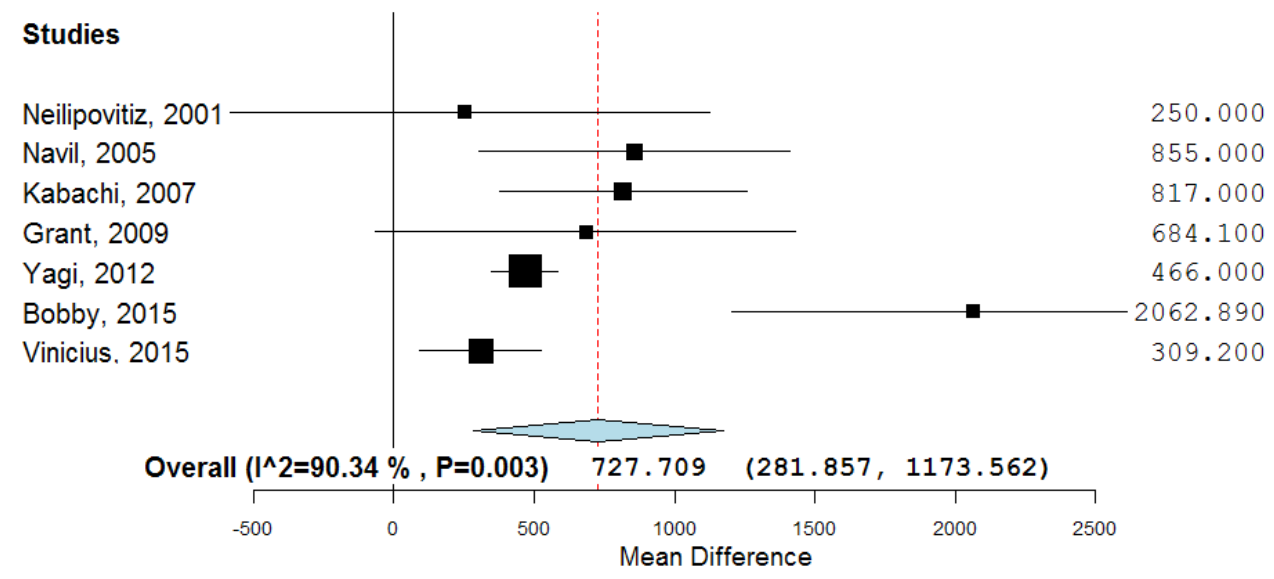

Figure 5 Forest plot displaying PL meta-analysis.

Table 3 Heterogeneity measures of blood loss

\begin{tabular}{llll}
\hline Source & Value & df & P value \\
\hline Cochrane Q & 20.05 & 6 & 0.001 \\
$I^{2}(\%)$ & 90.34 & - & - \\
\hline
\end{tabular}

and weight effect of the six studies included for blood loss. Figure 4 also summarizes the mean blood transfusion. As can seen in Table 3, all studies' profile for both TXA and control groups that included sample size, mean blood transfusion volume (BTV), effect size (MD), upper and lower limits of the CI were overlapped as observed in forest plot (Figure 6) and that indicated generalized homogeneity. The overall model results indicated that the effect size of BVT was $268.30 \mathrm{~mL}$, with CI ranged from 105.51 to $431.41 \mathrm{~mL}$, which was significant $(\mathrm{P}<0.001)$, implying that the use of TXA inducted substantial reduction in blood transfusion postoperatively. As observed from forest plot (Figure 6), an overlap among all the CI's of all studies exists. The observed scatter is due to sampling error, and the true effects of TXA in all studies are identical. This is also true as $\mathrm{I}^{2}=72.27 \%$ implies more than $72.27 \%$ of the proportion of blood transfusion reduction inducted by TXA, treatment effect (Table 5).

\section{Discussion}

This study is ultimately a step forward to generalize the use of tranexamic acid in spinal surgery, scoliosis reduction. The results showed positive outcomes and significant differences in terms of blood reduction and transfusions, potentially making TXA the drug of choice. Despite its effectiveness, studies on its prophylactic use in scoliosis surgery are limited. Questions arise on the role of TXA in perioperative blood reduction and postoperative allogenic transfusion. Limitations can be found in this study due to the inclusion of retrospective studies. However, similar benefits of blood loss reduction and transfusion under the use of TXA have been documented in various surgical specialities such as in total knee arthroplasty (21) and radical surgery (22) to that of spine surgeries. In addition, adverse effects play major role when selecting a drug. Neilipovitz et al. (14) evaluated the risks in a randomized-control trial revealing no cases of clinical thrombotic events or hemodynamic instability. Another study showed the safety of TXA use in scoliosis surgery upon postoperative examination detecting no clinical evidence of deep venous thrombosis (15). Subsequent to these two studies, numerous other studies support its perioperative safety as shown in the studies included in our meta-analysis (Table 6). Another point to add is the presence of co-morbidity. Patients with preexisting renal or hepatic disorders, bleeding diathesis, or coagulation abnormalities are most commonly excluded from studies due to their vulnerability and susceptibility to adverse events. On the other hand, safe use of TXA can be achieved in variant situations such as adjusting the dosage for patients with renal insufficiency (23).

When weighing the risks and benefits, dose regimens should be taken in consideration. Not having an established optimal dose can be quite challenging. In high dose versus low dose study, the group of patients on a loading dose of $50 \mathrm{mg} / \mathrm{kg}$ and a maintenance dose of $5 \mathrm{mg} / \mathrm{kg} / \mathrm{h}$ had a lower estimated blood loss than those who were on a loading 
Table 4 Studies profile, effect size, confidence intervals and weight for blood volume transfusion

\begin{tabular}{|c|c|c|c|c|c|c|c|c|}
\hline \multirow{2}{*}{ Studies } & \multicolumn{2}{|c|}{ Control } & \multicolumn{2}{|c|}{ TXA } & \multirow{2}{*}{ MD } & \multirow{2}{*}{ Lower } & \multirow{2}{*}{ Upper } & \multirow{2}{*}{ Weight (\%) } \\
\hline & $\mathrm{N}$ & BVT & $\mathrm{N}$ & BVT & & & & \\
\hline Neilipovitz, 2001 & 18.00 & $1,784.00$ & 22.00 & $1,253.00$ & 531.00 & 29.89 & $1,032.12$ & 7.78 \\
\hline Navil, 2005 & 21.00 & 940.00 & 23.00 & 615.00 & 325.00 & -35.06 & 685.06 & 12.06 \\
\hline Grant, 2009 & 15.00 & $1,373.00$ & 11.00 & 687.90 & 685.10 & 108.34 & $1,261.86$ & 6.28 \\
\hline Yagi, 2012 & 63.00 & 377.00 & 43.00 & 258.00 & 119.00 & 30.43 & 207.57 & 26.95 \\
\hline Bobby, 2015 & 35.00 & 891.00 & 55.00 & 472.50 & 418.50 & 264.78 & 572.22 & 23.23 \\
\hline Vinicius, 2015 & 19.00 & 176.30 & 21.00 & 111.10 & 65.20 & -81.13 & 211.53 & 23.69 \\
\hline
\end{tabular}

BVT, blood volume transfusion.

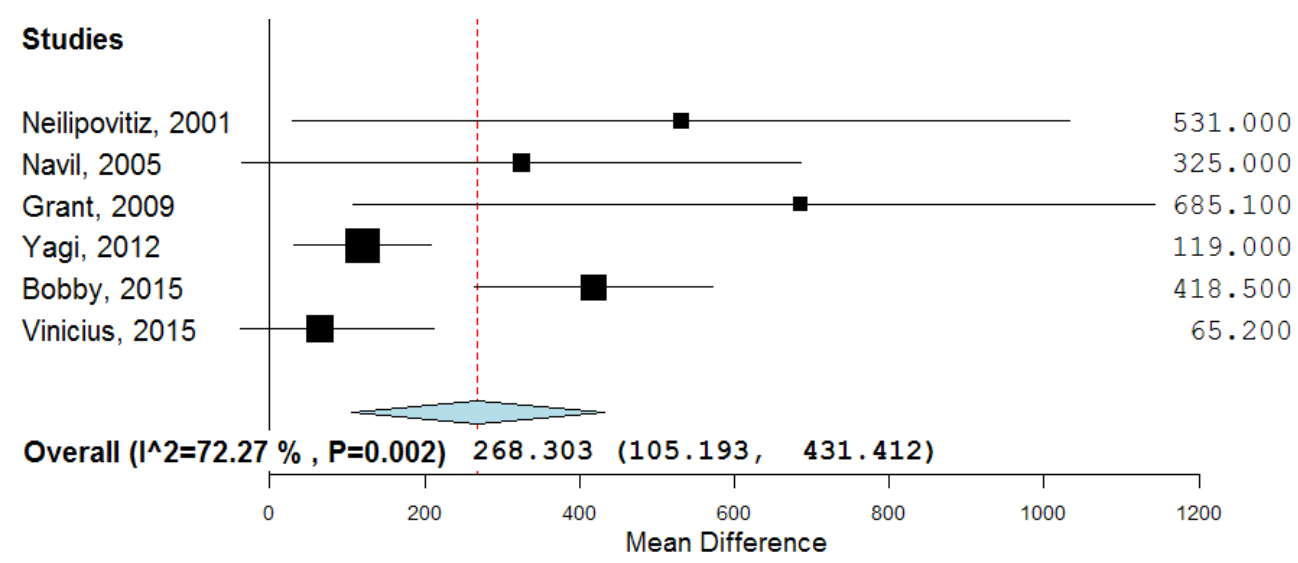

Figure 6 Forest plot displaying PL meta-analysis for blood transfusions.

Table 5 Heterogeneity measures of blood transfusion

\begin{tabular}{lccc}
\hline Source & Value & df & P value \\
\hline Cochrane Q & 18,87 & 5 & 0.001 \\
$\mathrm{I}^{2}(\%)$ & 72.27 & - & - \\
\hline
\end{tabular}

dose of $10 \mathrm{mg} / \mathrm{kg}$ and a maintenance dose of $1 \mathrm{mg} / \mathrm{kg} / \mathrm{h}$ by $\mathrm{P}=0.01$ difference (24). Nevertheless, high doses of a drug raise great concern regarding toxicity, and to which extent of increase can the drug safely be administered. In a TXA study of different dose regimens, Xie et al. (25) declared that a loading dose of $100 \mathrm{mg} / \mathrm{kg}$ with a maintenance dose of $10 \mathrm{mg} / \mathrm{kg} / \mathrm{h}$, double of which mentioned in the previous study, showed no signs of renal toxicity, seizures, DVT, or MI upon assessment. Conclusively, TXA is a safe drug though an optimum dose is greatly recommended for exploration. Further to be considered is the etiology of the
Table 6 Post-surgical risks

\begin{tabular}{ll}
\hline Studies & Post-surgical risk \\
\hline Neilipovitz, 2001 & Safe procedure \\
Navil, 2005 & Safe procedure \\
Kaabachi, 2007 & No data regarding risks \\
Grant, 2009 & No data regarding risks \\
Yagi, 2012 & No intraoperative complications \\
Bobby, 2015 & Safe procedure \\
Vinicius, 2015 & Not mentioned \\
\hline
\end{tabular}

disorder, secondary scoliosis, neuromuscular or congenital etiologies, manifested greater blood loss tendency than that of idiopathic etiology, though the mechanism behind the increase bleeding is undetermined (26). With such variables, study's results may be deviated. To illustrate, 
various measures should be taken in order to minimize the high amount of blood loss experienced in patients' with Duchenne Muscular Dystrophy undergoing posterior spinal fusion such as deliberate hypotension technique and patient positioning, yet with the use of TXA, minimal of $740 \mathrm{~mL}$ blood reduction and 2,135 $\mathrm{mL}$ maximum is to be expected (26). Which happens to make TXA the advanced management of neuromuscular scoliosis.

Moreover, hospitals' protocols on autologous blood transfusion, hemoglobin level, and the use of cell saver can bias the study's results. In Choi et al. (27) study on the effectiveness and safety of TXA in spinal deformity surgery, same blood transfusion guidelines were used for both TXA group and control group, allogenic transfusions were preformed to maintain hemoglobin level between 7-8 $\mathrm{mg} / \mathrm{dL}$. In some institutions, anesthesia protocols are standard in scoliosis surgery which leads to limitations in the study (17). As well, in randomized-control trials, patients' demographic data such as age, gender, body mass index, medical history vary between the two groups contributing to alteration in the results, but such differences are minor and of little or no clinical relevance. Furthermore, scoliosis surgery is based on medical criteria. Skeletal maturity, degree of curvature, and progression of the curvature are included when considering the surgery (28). However, patients as young as 8 years old may undergo idiopathic scoliosis surgery. Hence, introducing a drug for study in a vulnerable age group can be demanding leading to limited studies of TXA in spine surgery. More emphasis on its safety and significant efficacy on both patients and hospitals is crucial. According to the CRASH 2 trial (29), TXA administration is remarkably cost-effective in low, middle, high income settings directly, and indirectly by reducing the cost of additional hospital stays. In other circumstances, economical saving by decreasing the costs of treatment for allogenic transfusions' side-effects is promising.

When covering the intraoperative aspect of TXA use, surgeons' and anesthesiologists' preferences are taken into account. In surgery for instance, intraoperative transfusion is based on the surgical team's decision such when hemoglobin level reaches $7.0 \mathrm{~g} / \mathrm{dL}$ or less (30) and the TXA dose to be administered is under the anesthesiologist's preference. Neilipovitz et al. (14) study conducted a short questionnaire at the end of the surgery that was completed by the attending surgeon rating the level of surgical difficulty on a visual analogue scale of 0 (no difficulty) to 10 (extremely difficult) and the operative conditions in term of bleeding, 0 (negligible bleeding) to 10 (massive bleeding).
The results were similar for both TXA group $(n=22)$ and the control group $(n=18)(14)$. However, upon perspectives, results may respectfully fluctuate. One of the most significant advantages health practitioners will face with the use of TXA is the decline in number of cases of bloodborne infections and immunologic reactions associated with blood transfusions as such cases present to be challenging for surgeons. In our results, a notable reduction of blood transfusion was seen in patients treated with TXA that happens to make TXA a deal breaker when measuring the consequences. From anesthesia's point of view, it has been addressed that the use of TXA with regional and general anesthesia has no complications (31). In fact, the use of TXA with epidural anesthesia followed $65 \%$ of reduction in blood loss (31). It is clear that TXA plays a tremendous role in the decline of perioperative blood loss and allogenic transfusions. Thus, this meta-analysis will help guide future scoliosis management and further study exploration on its use in spine surgeries.

\section{Conclusions}

Although the number of patients in each of the included studies in this meta-analysis is relatively small, tranexamic acid has shown to effectively reduce blood loss and blood transfusion in idiopathic scoliosis correction surgery. Nevertheless, further multi-center double blinded randomized control trials involving a larger number of patients are required to confirm the efficacy and safety of tranexamic acid in idiopathic scoliosis surgery.

\section{Acknowledgements}

The authors would like to thank Dr. Abdulrahman AlArjani, Consultant Spine \& Head of Spine Department, Dr. Abdulwahid Barnawi, Consultant Spine \& Vice Head of Spine Department, Dr. Yasser Brikeet, Consultant Spine \& Pediatric Orthopedics Head of Spinal Deformity Unit, Dr. Abdullah Arab, Consultant Spine, from Prince Sultan Military Medical City for their invaluable support and expertize throughout this research.

\section{Footnote}

Conflicts of Interest: The authors have no conflicts of interest to declare.

\section{References}


1. Lonstein JE, Bradford DS, Winter RB, et al. Moe's textbook of scoliosis and other spinal deformities. $3 \mathrm{rd}$ ed. Philadelphia: WB Saunders, 1995:219-56.

2. Asher MA, Burton DC. A concept of idiopathic scoliosis deformities as imperfect torsion(s). Clin Orthop Relat Res 1999;(364):11-25.

3. Charles YP, Diméglio A, Marcoul M, et al. Influence of idiopathic scoliosis on three-dimensional thoracic growth. Spine (Phila Pa 1976) 2008;33:1209-18.

4. Mielke CH, Lonstein JE, Denis F, et al. Surgical treatment of adolescent idiopathic scoliosis. A comparative analysis. J Bone Joint Surg Am 1989;71:1170-7.

5. Kim C, Park SS, Davey JR. Tranexamic acid for the prevention and management of orthopedic surgical hemorrhage: current evidence. J Blood Med 2015;6:239-44.

6. Eubanks JD. Antifibrinolytics in major orthopaedic surgery. J Am Acad Orthop Surg 2010;18:132-8.

7. $\mathrm{Ng} W$, Jerath $\mathrm{A}, \mathrm{W}$ sowicz $M$. Tranexamic acid: a clinical review. Anaesthesiol Intensive Ther 2015;47:339-50.

8. Munoz JJ, Birkmeyer NJ, Birkmeyer JD, et al. Is epsilonaminocaproic acid as effective as aprotinin in reducing bleeding with cardiac surgery?: a meta-analysis. Circulation 1999;99:81-9.

9. Bailey AM, Baker SN, Weant KA. Tranexamic acid for trauma-related hemorrhage. Adv Emerg Nurs J 2014;36:123-31.

10. Yu X, Li W, Xu P, et al. Safety and Efficacy of Tranexamic Acid in Total Knee Arthroplasty. Med Sci Monit 2015;21:3095-103.

11. Danninger T, Memtsoudis SG. Tranexamic acid and orthopedic surgery-the search for the holy grail of blood conservation. Ann Transl Med 2015;3:77.

12. Zhang F, Wang K, Li FN, et al. Effectiveness of tranexamic acid in reducing blood loss in spinal surgery: a meta-analysis. BMC Musculoskelet Disord 2014;15:448.

13. Ialenti MN, Lonner BS, Verma K, et al. Predicting operative blood loss during spinal fusion for adolescent idiopathic scoliosis. J Pediatr Orthop 2013;33:372-6.

14. Neilipovitz DT, Murto K, Hall L, et al. A randomized trial of tranexamic acid to reduce blood transfusion for scoliosis surgery. Anesth Analg 2001;93:82-7.

15. Sethna NF, Zurakowski D, Brustowicz RM, et al. Tranexamic acid reduces intraoperative blood loss in pediatric patients undergoing scoliosis surgery. Anesthesiology 2005;102:727-32.

16. Kaabachi O, Koubaa W, Chaffii D, et al. Tranexamic acid reduces blood transfusion requirements in scoliosis surgery. Euro J Anesth 2007;24:67.
17. Grant JA, Howard J, Luntley J, et al. Perioperative blood transfusion requirements in pediatric scoliosis surgery: the efficacy of tranexamic acid. J Pediatr Orthop 2009;29:300-4.

18. Yagi M, Hasegawa J, Nagoshi N, et al. Does the intraoperative tranexamic acid decrease operative blood loss during posterior spinal fusion for treatment of adolescent idiopathic scoliosis? Spine 2012;37:E1336-42.

19. Ng B, Chau WW, Hung A, et al. Use of tranexamic acid (TXA) on reducing blood loss during scoliosis surgery in Chinese adolescents. Scoliosis 2015;10:28.

20. Rocha V, Barros A, Naves C, et al. Use of tranexamic acid for controlling bleeding in thoracolumbar scoliosis surgery with posterior instrumentation. Rev Bras Ortop 2015;50:226-31.

21. Hiippala ST, Strid LJ, Wennerstrand MI, et al. Tranexamic acid radically decreases blood loss and transfusions associated with total knee arthroplasty. Anesth Analg 1997;84:839-44.

22. Gupta K, Rastogi B, Krishan A, et al. The prophylactic role of tranexamic acid to reduce blood loss during radical surgery: A prospective study. Anesth Essays Res 2012;6:70-3.

23. Neilipovitz DT. Tranexamic acid for major spinal surgery. Eur Spine J 2004;13:S62-5.

24. Johnson DJ, Johnson CC, Goobie SM, et al. High-dose vs Low-dose Tranexamic Acid to Reduce Transfusion Requirements in Pediatric Scoliosis Surgery. J Pediatr Orthop 2017;37:e552-e7.

25. Xie JM, Li T, Wang Y, et al. Different Dose Regimens of Tranexamic Acid Reduces Perioperative Blood Loss and Blood Transfusion in Adolescent Idiopathic Scoliosis Patients: A Prospective, Randomized Control Study. Spine J 2015;15:S176.

26. Shapiro F, Zurakowski D, Sethna NF. Tranexamic acid diminishes intraoperative blood loss and transfusion in spinal fusions for duchenne muscular dystrophy scoliosis. Spine (Phila Pa 1976) 2007;32:2278-83.

27. Choi HY, Hyun SJ, Kim KJ, et al. Effectiveness and Safety of Tranexamic Acid in Spinal Deformity Surgery. J Korean Neurosurg Soc 2017;60:75-81.

28. Maruyama T, Takeshita K. Surgical treatment of scoliosis: a review of techniques currently applied. Scoliosis 2008;3:6.

29. Roberts I, Shakur H, Coats T, Hunt B, Balogun E, Barnetson L, et al. The CRASH-2 trial: a randomised controlled trial and economic evaluation of the effects of tranexamic acid on death, vascular occlusive events and transfusion requirement in bleeding trauma patients. Health Technol Assess 2013;17:1-79. 
30. Lykissas MG, Crawford AH, Chan G, et al. The effect of tranexamic acid in blood loss and transfusion volume in adolescent idiopathic scoliosis surgery: a single-surgeon experience. J Child Orthop 2013;7:245-9.

31. Ezhevskaya A, Presnyakova M, Prusakova Z. The Double-
Blind Study of Isolated and Combined use of Tranexamic Acid and Epidural Anesthesia in Scoliosis Surgery. Reg Anesth \& Pain Med 2015;40:5.

Cite this article as: Alajmi T, Saeed H, Alfaryan K, Alakeel A, Alfaryan T. Efficacy of tranexamic acid in reducing blood loss and blood transfusion in idiopathic scoliosis: a systematic review and meta-analysis. J Spine Surg 2017;3(4):531-540. doi: 10.21037/jss.2017.08.17 